\begin{tabular}{ll} 
C O L L O Q U I U M & M A T H E M A T I C U M \\
\hline Vol. LXVIII & 1995 \\
\hline
\end{tabular}

ON MÜNTZ RATIONAL APPROXIMATION IN MULTIVARIABLES

BY

S. P. ZHOU (EDMONTON, ALBERTA)

The present paper shows that for any $s$ sequences of real numbers, each with infinitely many distinct elements, $\left\{\lambda_{n}^{j}\right\}, j=1, \ldots, s$, the rational combinations of $x_{1}^{\lambda_{m_{1}}^{1}} x_{2}^{\lambda_{m_{2}}^{2}} \ldots x_{s}^{\lambda_{m_{s}}^{s}}$ are always dense in $C_{I^{s}}$.

1. Introduction. Let $C_{[0,1]}$ be the class of all real continuous functions in $[0,1]$. For $f \in C_{[0,1]}$,

$$
\begin{gathered}
\omega(f, t)=\max _{0<h<t, x \in[0,1-h]}|f(x+h)-f(x)|, \\
\|f\|=\max _{x \in[0,1]}|f(x)| .
\end{gathered}
$$

Given a subspace $S$ of $C_{[0,1]}$, let

$$
R(S)=\{P(x) / Q(x): P(x) \in S, Q(x) \in S, Q(x)>0, x \in(0,1]\},
$$

where we assume that $\lim _{x \rightarrow 0+} P(x) / Q(x)=P(0) / Q(0)$ is finite in the case $Q(0)=0$. For a sequence of real numbers $\Lambda=\left\{\lambda_{n}\right\}_{n=0}^{\infty}$, write

$$
R(\Lambda)=R\left(\operatorname{span}\left\{x^{\lambda_{n}}\right\}\right)
$$

From Müntz's theorem (cf. [2]), it is well-known that the combinations of $x^{\lambda_{n}}$ for

$$
0=\lambda_{0}<\lambda_{1}<\lambda_{2}<\ldots
$$

are dense in $C_{[0,1]}$ if and only if

$$
\sum_{n=1}^{\infty} \frac{1}{\lambda_{n}}=\infty .
$$

As to the rational case, in 1976, Somorjai [6] showed a beautiful result that under (1), $R(\Lambda)$ is always dense in $C_{[0,1]}$. In 1978, Bak and Newman [1] proved that if $\lambda_{n}$ is a sequence of distinct positive numbers, then $R(\Lambda)$ is dense in $C_{[0,1]}$ as well. Recently, our work [7] showed that the above result

1991 Mathematics Subject Classification: 41A20, 41A30, 41A63. 
also holds for any sequence of real numbers with infinitely many distinct elements.

On the other hand, S. Ogawa and K. Kitahara [5] gave a generalization of Müntz's theorem to multivariable cases. They proved $\left({ }^{1}\right)$ that for two given positive monotone sequences $\left\{\alpha_{i}\right\},\left\{\beta_{j}\right\}$, the set $\{1\} \cup\left\{x^{\alpha_{i}}\right\} \cup\left\{y^{\beta_{j}}\right\}$ is complete in $C_{I^{2}}$ if and only if $\sum_{i=1}^{\infty} 1 / \alpha_{i}$ and $\sum_{j=1}^{\infty} 1 / \beta_{j}$ diverge, where

$$
I^{s}=\left\{X=\left(x_{1}, \ldots, x_{s}\right): 0 \leq x_{j} \leq 1,1 \leq j \leq s\right\},
$$

and $C_{I^{s}}$ is the class of all continuous functions on $I^{s}$.

For many reasons, it is quite reasonable to conjecture that the conclusion corresponding to that of [7] will hold for Müntz rational approximation in the multivariable case, that is, for any $s$ sequences of real numbers $\left\{\lambda_{n}^{j}\right\}$, $j=1, \ldots, s$, each with infinitely many distinct elements, the rational combinations of $\left\{x_{1}^{\lambda_{m_{1}}^{1}} x_{2}^{\lambda_{m_{2}}^{2}} \ldots x_{s}^{\lambda_{m_{s}}^{s}}\right\}$ are always dense in $C_{I^{s}}$. Since rational combinations are not linear, it is not a trivial work.

The present paper will prove that this is true.

\section{Result and proof}

ThEOREM. Let $\Lambda^{j}=\left\{\lambda_{n}^{j}\right\}, j=1, \ldots, s$, be s sequences of real numbers, each with infinitely many distinct elements. Then $R\left(\Lambda^{1} \times \ldots \times \Lambda^{s}\right)$ is dense in $C_{I^{s}}$.

We need the following lemmas from the univariable case, the first two of which are due to Somorjai [6] and the author [7]. We will, however, give the sketch of proofs here for the sake of completeness.

LEMMA 1 (Somorjai [6]). Let $\left\{\lambda_{n}\right\}$ be a sequence of real numbers such that $\lambda_{n} \rightarrow+\infty$ as $n \rightarrow \infty$. Given $N \geq 1$, for any $f \in C_{[0,1]}$, there are an integer $n_{N}$ and an operator

$$
\sum_{k=0}^{N} f\left(\frac{k}{N}\right) \frac{Z_{k}(x)}{Z(x)}=: r_{N}^{1}(f, x) \in R\left(\left\{\lambda_{j}\right\}_{j=0}^{n_{N}}\right)
$$

with

such that

$$
0 \leq Z_{k}(x) \in \operatorname{span}\left\{x^{\lambda_{j}}\right\}_{j=0}^{n_{N}}, \quad Z(x)=\sum_{k=0}^{N} Z_{k}(x)
$$

$$
\left\|f-r_{N}^{1}(f)\right\|=O\left(\omega\left(f, N^{-1}\right)\right) .
$$

Proof. We select a sequence $\left\{\lambda_{n_{j}}\right\}_{j=1}^{n_{N}}$ from $\Lambda$ by induction. Let $\lambda_{n_{0}}$ be any element from $\Lambda$, and $Z_{0}(x)=x^{\lambda_{n_{0}}}$. Choose $\lambda_{n_{j+1}}$ with the following properties:

$\left({ }^{1}\right)$ For convenience, we only state their result for two variables. 


$$
\begin{gathered}
Z_{j+1}(x)=\left(\frac{N}{j+1} x\right)^{\lambda_{n_{j+1}}} \leq N^{-1} Z_{j}(x) \quad \text { for } x<\frac{j}{N}, \\
Z_{j+1}(x)>N Z_{j}(x) \quad \text { for } x>\frac{j+2}{N} .
\end{gathered}
$$

Define

$$
r_{N}^{1}(f, x)=\sum_{k=0}^{N} f\left(\frac{k}{N}\right) \frac{Z_{k}(x)}{\sum_{v=0}^{N} Z_{v}(x)}
$$

for $f \in C_{[0,1]}$. Then by calculation

$$
f(x)-r_{N}^{1}(f, x)=O\left(\omega\left(f, N^{-1}\right)\right) .
$$

LEMma 2 (Zhou [7]). Let $\left\{\lambda_{n}\right\}$ be a sequence of real numbers such that $\lambda_{n} \rightarrow-\infty$ as $n \rightarrow \infty$. Given $N \geq 1$, for any $f \in C_{[0,1]}$, there are an integer $n_{N}$ and an operator

$$
\sum_{k=0}^{N} f\left(\frac{k}{N}\right) \frac{C_{k}(x)}{C(x)}=: r_{N}^{2}(f, x) \in R\left(\left\{\lambda_{j}\right\}_{j=0}^{n_{N}}\right)
$$

with

such that

$$
0 \leq C_{k}(x) \in \operatorname{span}\left\{x^{\lambda_{j}}\right\}_{j=0}^{n_{N}}, \quad C(x)=\sum_{k=0}^{N} C_{k}(x)
$$

$$
\left\|f-r_{N}^{2}(f)\right\|=O\left(\omega\left(f, N^{-1}\right)\right) .
$$

Pro of. Similar to Lemma 1 , let $\lambda_{n_{1}}$ be any element from $\Lambda$, and $C_{1}^{*}(x)=$ $x^{\lambda_{n_{1}}}$. Choose $\lambda_{n_{j+1}}$ satisfying

$$
\begin{gathered}
C_{j+1}^{*}(x)=\left(\frac{N}{N-j} x\right)^{\lambda_{n_{j+1}}} \geq N C_{j}^{*}(x) \quad \text { for } x<\frac{N-j-1}{N}, \\
C_{j+1}^{*}(x)<N^{-1} C_{j}^{*}(x) \quad \text { for } x>\frac{N-j+2}{N} .
\end{gathered}
$$

For $f \in C_{[0,1]}$, define

$$
r_{N}^{2}(f, x)=\sum_{k=1}^{N} f\left(\frac{N-k+1}{N}\right) \frac{C_{k}^{*}(x)}{\sum_{v=1}^{N} C_{v}^{*}(x)} .
$$

Then the required result follows.

Lemma 3. Let $\left\{\lambda_{n}\right\}$ be a sequence of real numbers with infinitely many distinct elements such that $\lambda_{n} \rightarrow l$ as $n \rightarrow \infty$ with $-\infty<l<\infty$. Given $N \geq 1$ and $\varepsilon>0$, there are an integer $n_{N}$ and an operator

$$
\sum_{k=0}^{N} f\left(e^{1-N / k}\right) \frac{D_{k}(x)}{D(x)}=: r_{N}^{3}(f, x) \in R\left(\left\{\lambda_{j}\right\}_{j=0}^{n_{N}}\right)
$$


with $D_{k}(x), D(x) \in \operatorname{span}\left\{x^{\lambda_{j}}\right\}_{j=0}^{n_{N}}$ such that

$$
\left\|f-r_{N}^{3}(f)\right\| \leq 2 \omega\left(g, N^{-1 / 2}\right)+\|f\| \varepsilon,
$$

where $g(u)=f\left(e^{1-1 / u}\right)$. Precisely, we have

$$
\begin{gathered}
\frac{D_{k}(x)}{D(x)}=G_{k}(x)+H_{k}(x) \\
G_{k}(x)=\left(\begin{array}{c}
N \\
k
\end{array}\right)\left(\frac{-1}{\ln (x / e)}\right)^{k}\left(1+\frac{1}{\ln (x / e)}\right)^{N-k},
\end{gathered}
$$

and

$$
\left|H_{k}(x)\right| \leq \frac{\varepsilon}{N+1}
$$

Proof. There are two possibilities: (i) There is a subsequence $\left\{\lambda_{n_{k}}\right\}$ of $\left\{\lambda_{n}\right\}$ which strictly increases to $\lambda<+\infty$ (in symbols $\lambda_{n_{k}} \nearrow \lambda<+\infty$ ) as $k \rightarrow \infty$; (ii) there is a subsequence $\left\{\lambda_{n_{k}}\right\}$ which strictly decreases to $\lambda>-\infty$ (in symbols $\lambda_{n_{k}} \searrow \lambda>-\infty$ ) as $k \rightarrow \infty$. We will prove Lemma 3 in these two cases separately.

Case (i). For convenience, we still write $\lambda_{n_{k}}$ as $\lambda_{n}$. So under the hypothesis, $\lambda_{n} \nearrow \lambda<+\infty$ as $n \rightarrow \infty$. Let $\alpha_{0}<\alpha_{1}<\ldots$, and let $P_{k}(x)$ denote the $k$ th divided difference of $(x / e)^{\alpha}$ at $\alpha=\alpha_{2 N-1}, \alpha_{2 N-2}, \ldots, \alpha_{2 N-k-1}$ for $k=0,1, \ldots, N-1$, that is,

$$
\begin{aligned}
& P_{0}(x)=P_{0}\left(x, \alpha_{2 N-1}\right)=(x / e)^{\alpha_{2 N-1}} \\
& P_{1}(x)=P_{1}\left(x, \alpha_{2 N-1}, \alpha_{2 N-2}\right)=\frac{(x / e)^{\alpha_{2 N-1}}-(x / e)^{\alpha_{2 N-2}}}{\alpha_{2 N-1}-\alpha_{2 N-2}},
\end{aligned}
$$

in general,

$$
\begin{aligned}
& P_{k}(x)=P_{k}\left(x, \alpha_{2 N-1}, \ldots, \alpha_{2 N-k-1}\right) \\
&=\frac{P_{k-1}\left(x, \alpha_{2 N-1}, \ldots, \alpha_{2 N-k}\right)-P_{k-1}\left(x, \alpha_{2 N-2}, \ldots, \alpha_{2 N-k-1}\right)}{\alpha_{2 N-1}-\alpha_{2 N-k-1}}, \\
& 0 \leq k \leq N-1,
\end{aligned}
$$

and

$$
P_{N}(x)=P_{N}\left(x, \alpha_{N}, \ldots, \alpha_{0}\right) .
$$

By the mean value theorem

(5) $\quad P_{k}(x)=\frac{(x / e)^{\eta_{k}} \ln ^{k}(x / e)}{k !}$,

$$
\alpha_{2 N-k-1} \leq \eta_{k} \leq \alpha_{2 N-1}, k=0,1, \ldots, N-1,
$$

(6) $\quad P_{N}(x)=\frac{(x / e)^{\eta_{N}} \ln ^{N}(x / e)}{N !}, \quad \alpha_{0} \leq \eta_{N} \leq \alpha_{N}$. 
Now let $f \in C_{[0,1]}$. Then $g(u)=f\left(e^{1-1 / u}\right) \in C_{[0,1]}$. Write

$$
\begin{aligned}
B_{N}(f, x) & =\sum_{k=0}^{N} f\left(\frac{k}{N}\right)\left(\begin{array}{l}
N \\
k
\end{array}\right) x^{k}(1-x)^{N-k} \\
& =\sum_{k=0}^{N} f\left(\frac{k}{N}\right)\left(\begin{array}{c}
N \\
k
\end{array}\right) \sum_{j=0}^{N-k}(-1)^{j}\left(\begin{array}{c}
N-k \\
j
\end{array}\right) x^{j+k} \\
& =\sum_{k=0}^{N} f\left(\frac{k}{N}\right)\left(\begin{array}{l}
N \\
k
\end{array}\right) \sum_{j=k}^{N}(-1)^{j-k}\left(\begin{array}{c}
N-k \\
j-k
\end{array}\right) x^{j} .
\end{aligned}
$$

For given $N \geq 1$, the well-known Bernstein theorem implies that

$$
\left\|g(u)-B_{N}(g, u)\right\|<\frac{3}{2} \omega\left(g, N^{-1 / 2}\right)
$$

that is,

$$
\left\|f(x)-B_{N}(g,-1 / \ln (x / e))\right\|<\frac{3}{2} \omega\left(g, N^{-1 / 2}\right) .
$$

Choose sufficiently large $m$ such that for $k \geq m$,

$$
0<\lambda-\lambda_{k}<\varepsilon /\left(4^{N}(N+1)\right) .
$$

Set $\alpha_{k}=\lambda_{m+k}, k=0,1, \ldots, 2 N-1$. Define

$$
r_{N}^{3}(f, x)=\sum_{k=0}^{N} f\left(e^{1-N / k}\right)\left(\begin{array}{c}
N \\
k
\end{array}\right) \frac{\sum_{j=k}^{N}(-1)^{2 j-k}(N-j) !\left(\begin{array}{c}
N-k \\
j-k
\end{array}\right) P_{N-j}(x)}{N ! P_{N}(x)} .
$$

Then $r_{N}^{3}(f, x)$ is a rational combination of $\left\{x^{\lambda_{j}}\right\}_{j=m}^{m+2 N-1}$, and by (5), (6),

$$
r_{N}^{3}(f, x)=\sum_{k=0}^{N} f\left(e^{1-N / k}\right)\left(\begin{array}{c}
N \\
k
\end{array}\right) \sum_{j=k}^{N}(-1)^{j-k}\left(\begin{array}{c}
N-k \\
j-k
\end{array}\right)\left(\frac{-1}{\ln (x / e)}\right)^{j}(x / e)^{\eta_{j}^{*}}
$$

with $\eta_{0}^{*}=0,0<\eta_{j}^{*} \leq \lambda_{m+N}-\lambda_{m} \leq \lambda-\lambda_{m}, j=1, \ldots, N$. Now write

$$
\begin{aligned}
\sum_{j=k}^{N}(-1)^{j-k}\left(\begin{array}{c}
N-k \\
j-k
\end{array}\right)\left(\frac{-1}{\ln (x / e)}\right)^{j}(x / e)^{\eta_{j}^{*}} & \sum_{j=k}^{N}(-1)^{j-k}\left(\begin{array}{c}
N-k \\
j-k
\end{array}\right)\left(\frac{-1}{\ln (x / e)}\right)^{j} \\
& +\sum_{j=k}^{N}(-1)^{j-k}\left(\begin{array}{c}
N-k \\
j-k
\end{array}\right)\left(\frac{-1}{\ln (x / e)}\right)^{j}\left((x / e)^{\eta_{j}^{*}}-1\right) \\
= & \left(\frac{-1}{\ln (x / e)}\right)^{k}\left(1+\frac{1}{\ln (x / e)}\right)^{N-k}+\Sigma_{1} .
\end{aligned}
$$


Since for $\eta>0$,

we have

$$
\left\|\frac{1-(x / e)^{\eta}}{\ln (x / e)}\right\| \leq \eta
$$

for $k \geq 1$. Consequently,

$$
\left\|\frac{1-(x / e)^{\eta_{k}^{*}}}{\ln ^{k}(x / e)}\right\| \leq \eta_{k}^{*} \leq \frac{\varepsilon}{4^{N}(N+1)}
$$

$$
\left|\Sigma_{1}\right| \leq 4^{-N}(N+1)^{-1} \varepsilon \sum_{j=k}^{N}\left(\begin{array}{c}
N-k \\
j-k
\end{array}\right) \leq \frac{\varepsilon}{2^{N}(N+1)},
$$

and thus (2)-(4) are proved. Now from (4), (7), together with $H_{k}(x)=$ $\left(\begin{array}{l}N \\ k\end{array}\right) \Sigma_{1}$

$$
\begin{aligned}
\left\|f(x)-r_{N}^{3}(f, x)\right\| & \leq\left\|f(x)-B_{N}(g,-1 / \ln (x / e))\right\|+\|f\| \sum_{k=0}^{N}\left|H_{k}(x)\right| \\
& \leq \frac{3}{2} \omega\left(g, N^{-1 / 2}\right)+\|f\| \varepsilon,
\end{aligned}
$$

that is, Lemma 3 holds true in Case (i).

Case (ii). We may assume that $\lambda_{n} \searrow \lambda>-\infty$ as $n \rightarrow \infty$. Take

$$
\begin{aligned}
P_{k}(x) & =P_{k}\left(x, \lambda_{m}, \ldots, \lambda_{m+k}\right), \quad 0 \leq k \leq N-1, \\
P_{N}(x) & =P_{N}\left(x, \lambda_{m+N-1}, \ldots, \lambda_{m+2 N-1}\right),
\end{aligned}
$$

and

$$
r_{N}^{3}(f, x)=\sum_{k=0}^{N} f\left(e^{1-N / k}\right)\left(\begin{array}{c}
N \\
k
\end{array}\right) \frac{\sum_{j=k}^{N}(-1)^{2 j-k}(N-j) !\left(\begin{array}{c}
N-k \\
j-k
\end{array}\right) P_{N-j}(x)}{N ! P_{N}(x)} .
$$

Similar to Case (i), for given $\varepsilon>0$ and $N \geq 1$, we can prove (2)-(4) and for sufficiently large $m$,

$$
\left\|f(x)-r_{N}^{3}(f, x)\right\| \leq \frac{3}{2} \omega\left(g, N^{-1 / 2}\right)+\|f\| \varepsilon .
$$

The proof of Lemma 3 is now complete.

Proof of the Theorem. Given a sequence with infinitely many distinct elements $\left\{\lambda_{n}\right\}$, there are three possibilities: (i) $\left\{\lambda_{n}\right\}$ has at least one finite cluster point; (ii) one cluster point of $\left\{\lambda_{n}\right\}$ is $+\infty$; (iii) one cluster point of $\left\{\lambda_{n}\right\}$ is $-\infty$. Without loss of generality, we may assume

and

$$
\begin{array}{ll}
\lambda_{n}^{j} \rightarrow+\infty & \text { as } \quad n \rightarrow \infty, 1 \leq j \leq r, \\
\lambda_{n}^{j} \rightarrow-\infty & \text { as } \quad n \rightarrow \infty, r+1 \leq j \leq t,
\end{array}
$$

$$
\lambda_{n}^{j} \rightarrow l \quad \text { as } n \rightarrow \infty, t+1 \leq j \leq s,-\infty<l<+\infty .
$$


For given $N \geq 1$ and $\varepsilon>0$, from Lemmas 1-3, we may select a common $n_{N}$ such that

$$
\begin{array}{ll}
\left\|f(X)-r_{N}^{1}\left(f, x_{j}\right)\right\|=O\left(\omega_{x_{j}}\left(f, N^{-1}\right)\right), & 1 \leq j \leq r \\
\left\|f(X)-r_{N}^{2}\left(f, x_{j}\right)\right\|=O\left(\omega_{x_{j}}\left(f, N^{-1}\right)\right), & r+1 \leq j \leq t
\end{array}
$$

and

$$
\left\|f(X)-B_{N}\left(g,-1 / \ln \left(x_{j} / e\right)\right)\right\|=O\left(\omega_{x_{j}}\left(g, N^{-1 / 2}\right)\right), \quad t+1 \leq j \leq s,
$$

hold at the same time, where

$\omega_{x_{j}}(f, \delta)$

$$
=\max _{0 \leq h \leq \delta}\left|f\left(x_{1}, \ldots, x_{j}+h, x_{j+1}, \ldots, x_{s}\right)-f\left(x_{1}, \ldots, x_{j}, x_{j+1}, \ldots, x_{s}\right)\right| .
$$

Define

$$
\begin{aligned}
r_{N}(X)= & \sum_{0 \leq j_{1} \leq N} \ldots \sum_{0 \leq j_{s} \leq N} f\left(\frac{j_{1}}{N}, \ldots, \frac{j_{t}}{N}, \ldots, e^{1-N / j_{t+1}}, \ldots, e^{1-N / j_{s}}\right) \\
& \times \frac{Z_{j_{1}}\left(x_{1}\right)}{Z\left(x_{1}\right)} \ldots \frac{Z_{j_{r}}\left(x_{r}\right)}{Z\left(x_{r}\right)} \frac{C_{j_{r+1}}\left(x_{r+1}\right)}{C\left(x_{r+1}\right)} \ldots \frac{C_{j_{t}}\left(x_{t}\right)}{C\left(x_{t}\right)} \\
& \times \frac{D_{j_{t+1}}\left(x_{t+1}\right)}{D\left(x_{t+1}\right)} \ldots \frac{D_{j_{s}}\left(x_{s}\right)}{D\left(x_{s}\right)} .
\end{aligned}
$$

Evidently,

$$
r_{N}(X) \in R\left(\operatorname{span}\left\{x^{\lambda_{i}^{1}}\right\}_{i=0}^{n_{N}} \times \operatorname{span}\left\{x^{\lambda_{i}^{2}}\right\}_{i=0}^{n_{N}} \times \ldots \times \operatorname{span}\left\{x^{\lambda_{i}^{s}}\right\}_{i=0}^{n_{N}}\right) .
$$

From (2),

$$
\begin{aligned}
f(X)- & r_{N}(X) \\
= & \sum_{0 \leq j_{1} \leq N} \ldots \sum_{0 \leq j_{s} \leq N}\left(f(X)-f\left(\frac{j_{1}}{N}, \ldots, \frac{j_{t}}{N}, e^{1-N / j_{t+1}}, \ldots, e^{1-N / j_{s}}\right)\right) \\
& \times \frac{Z_{j_{1}}\left(x_{1}\right)}{Z\left(x_{1}\right)} \ldots \frac{Z_{j_{r}}\left(x_{r}\right)}{Z\left(x_{r}\right)} \frac{C_{j_{r+1}}\left(x_{r+1}\right)}{C\left(x_{r+1}\right)} \ldots \frac{C_{j_{t}}\left(x_{t}\right)}{C\left(x_{t}\right)} \\
& \times G_{j_{t+1}}\left(x_{t+1}\right) \ldots G_{j_{s}}\left(x_{s}\right)+\Sigma_{3}:=\Sigma_{2}+\Sigma_{3},
\end{aligned}
$$

where by Lemma 3,

(8)

$$
\left|\Sigma_{3}\right| \leq 4^{s}\|f\| \varepsilon
$$

Because $f(X) \in C_{I^{s}}$, there is a $\delta>0$ such that for $|X-Y|=$ $\sqrt{\sum_{j=1}^{s}\left(x_{j}-y_{j}\right)^{2}}<\delta$

$$
|f(X)-f(Y)|<\varepsilon
$$


while for $|X-Y| \geq \delta$,

$$
|f(X)-f(Y)| \leq 2 \delta^{-2}\|f\| \sum_{j=1}^{s}\left(x_{j}-y_{j}\right)^{2},
$$

therefore in any case

$$
|f(X)-f(Y)| \leq \varepsilon+2 \delta^{-2}\|f\| \sum_{j=1}^{s}\left(x_{j}-y_{j}\right)^{2} .
$$

Now

$$
\begin{aligned}
\left|\Sigma_{2}\right| \leq & \varepsilon+2 \delta^{-2}\|f\|\left(\sum_{0 \leq j_{1} \leq N} \ldots \sum_{0 \leq j_{s} \leq N} \sum_{i=1}^{t}\left(x_{i}-\frac{j_{i}}{N}\right)^{2}\right. \\
& \times \sum_{i=t+1}^{s}\left(x_{i}-e^{1-N / j_{i}}\right)^{2} \frac{Z_{j_{1}}\left(x_{1}\right)}{Z\left(x_{1}\right)} \ldots \frac{Z_{j_{r}}\left(x_{r}\right)}{Z\left(x_{r}\right)} \\
& \left.\times \frac{C_{j_{r+1}}\left(x_{r+1}\right)}{C\left(x_{r+1}\right)} \ldots \frac{C_{j_{t}}\left(x_{t}\right)}{C\left(x_{t}\right)} G_{j_{t+1}}\left(x_{t+1}\right) \ldots G_{j_{s}}\left(x_{s}\right)\right) \\
= & +2 \delta^{-2}\|f\|\left(\sum_{i=1}^{r} \sum_{j_{i}=0}^{N}\left(x_{i}-\frac{j_{i}}{N}\right)^{2} \frac{Z_{j_{i}}\left(x_{i}\right)}{Z\left(x_{i}\right)}\right. \\
& \left.\times \sum_{i=r+1}^{t} \sum_{j_{i}=0}^{N}\left(x_{i}-\frac{j_{i}}{N}\right)^{2} \frac{C_{j_{i}}\left(x_{i}\right)}{C\left(x_{i}\right)} \sum_{i=t+1}^{s} \sum_{j_{i}=0}^{N}\left(x_{i}-e^{1-N / j_{i}}\right)^{2} G_{j_{i}}\left(x_{i}\right)\right) .
\end{aligned}
$$

Noting that

$\sum_{k=0}^{N}\left(x-\frac{k}{N}\right)^{2} \frac{Z_{k}(x)}{Z(x)}=2 x\left(x-\sum_{k=0}^{N} \frac{k}{N} \frac{Z_{k}(x)}{Z(x)}\right)-\left(x^{2}-\sum_{k=0}^{N}\left(\frac{k}{N}\right)^{2} \frac{Z_{k}(x)}{Z(x)}\right)$,

from Lemma 1 we deduce that

$$
\sum_{k=0}^{N}\left(x-\frac{k}{N}\right)^{2} \frac{Z_{k}(x)}{Z(x)}=O\left(N^{-1 / 2}\right) .
$$

The same results also hold for $\sum_{k=0}^{N}(x-k / N)^{2} C_{k}(x) / C(x)$ and for $\sum_{k=0}^{N}\left(x-e^{1-N / k}\right)^{2} G_{k}(x)$ by applying Lemmas 2 and 3 . Altogether we have

$$
\left|\Sigma_{2}\right| \leq \varepsilon+2 \delta^{-2}\|f\| O\left(N^{-1 / 2}\right)
$$

thus combining (8) and (9) we get

$$
\left|f(X)-r_{N}(X)\right|=O(\varepsilon)+O\left(N^{-1 / 2}\right),
$$

which is the required result. 


\section{REFERENCES}

[1] J. Bak and D. J. Newman, Rational combinations of $x^{\lambda_{k}}, \lambda_{k} \geq 0$ are always dense in $C_{[0,1]}$, J. Approx. Theory 23 (1978), 155-157.

[2] E. W. Cheney, Introduction to Approximation Theory, McGraw-Hill, 1966.

[3] G. G. Lorentz, Bernstein Polynomials, Toronto, 1953.

[4] D. J. Newman, Approximation with Rational Functions, Amer. Math. Soc., Providence, R.I., 1978.

[5] S. Ogawa and K. Kitahara, An extension of Müntz's theorem in multivariables, Bull. Austral. Math. Soc. 36 (1987), 375-387.

[6] G. Somorjai, A Müntz-type problem for rational approximation, Acta Math. Acad. Sci. Hungar. 27 (1976), 197-199.

[7] S. P. Zhou, On Müntz rational approximation, Constr. Approx. 9 (1993), 435-444.

DEPARTMENT OF MATHEMATICS

UNIVERSITY OF ALBERTA

EDMONTON, ALBERTA

CANADA T6G 2G1

Reçu par la Rédaction le 18.8.1993;

en version modifiée le 30.3.1994 